\title{
Heating and Cooling of Hot Accretion Flows by Non Local Radiation
}

\author{
Ann A. Esin
}

Harvard-Smithsonian Center for Astrophysics, Cambridge MA 02138

\begin{abstract}
Hot optically thin accretion flow solutions are often used to model X-ray binaries and active galactic nuclei. Recently two new classes of advection-dominated models have been proposed: a two-temperature solution (Narayan \& Yi 1995, ApJ, 452, 710; Abramowicz et al. 1995, ApJ, 438, L37), and a one-temperature solution (Esin et al. 1996, ApJ, 465,312 ). Though detailed numerical spectra of advection-dominated flows include global radiative transfer effects (e.g. Narayan 1996, ApJ, $462,136)$, analytical calculations of the physical properties of these accretion solutions generally ignore the effects of non-local radiative transfer (e.g. Narayan \& Yi 1995; Esin et al. 1996). However, the optical depth for electron scattering in such flows is generally much lower than unity, and therefore, radiation emitted at one radius can potentially heat or cool the gas at other radii through Compton scattering. In this paper we investigate the importance of this effect. We discuss three situations:

1. Radiation from the inner regions of an advection-dominated flow Compton cooling gas at intermediate radii and Compton heating gas at large radii.
\end{abstract}

2. Soft radiation from an outer thin accretion disk Compton cooling a hot one- or two-temperature flow on the inside.

3. Soft radiation from an inner thin accretion disk Compton cooling hot gas in a surrounding one-temperature flow.

We describe how previous results are modified by these non-local interactions. We find that Compton heating or cooling of the gas by the radiation emitted in the inner regions of a hot flow is not important. Likewise, Compton cooling by the soft photons from an outer thin disk is negligible when the transition from a cold to a hot flow occurs at a radius greater than some minimum $R_{\mathrm{tr}, \min } \sim 10^{2.8}\left(\dot{M} / \dot{M}_{E d d}\right)^{3.5} \alpha^{-7} R_{S c h w}$. However, if the hot flow terminates at $R<R_{\mathrm{tr} \text {,min }}$, non-local cooling of the hot gas near the transition radius is so strong that the flow is cooled to a thin disk configuration. As a result, the transition radius decreases and the hot gas at the new boundary experiences even stronger external cooling. The resulting runaway process continues until the entire hot flow assumes the thin disk configuration. In the case of a thin disk surrounded by a hot one-temperature flow, we find that Compton cooling by soft radiation dominates over local cooling in the hot gas for $\dot{M} \gtrsim 10^{-3} \alpha \dot{M}_{E d d}$. As a result, the maximum accretion rate for which an advection-dominated one-temperature solution exists, decreases by a factor of $\sim 10$, compared to the value computed under an assumption of local energy balance. 